\title{
EP-142
}

\section{Microincision of the cyst duct is safe and effective for the failed laparoscopic transcystic common bile duct exploration}

\author{
Jiegao ZHU, Wei GUO* \\ Department of General Surgery, Beijing Friendship Hospital, Capital Medical University, Beijing, China
}

Introduction: Microincision of the cystic duct and its confluence part, which facilitated the application of transcystic-laparoscopic common bile duct (CBD) exploration (transcystic-LCBDE), had been described in some reports. However, it is unclear whether transcystic-LCBDE with microincision (micro-LCBDE) is effective and safe as transductal-LCBDE for the failed transcystic-LCBDE in patients with choledocholithiasis.

Methods: In this retrospective cohort study, we assigned patients with cholecystocholedocholithiasis to undergo transcystic-LCBDE and laparoscopic cholecystectomy. The clinical outcomes of one-to-one propensity-matched pairs of Micro-LCBDE and transductal-LCBDE groups were compared.

Results: Between January 2007 and December 2018, 1650 patients with confirmed choledocholithiasis attempted transcystic-LCBDE. Micro-LCBDE was done successfully in 128 patients (7.8\%) and transductal-LCBDE in 69 patients (4.2\%). By one-to-one propensity score matching, 68 pairs were selected. There was no significant difference in in-hospital complication rate: $8.8 \%$ in micro-LCBDE group and $11.8 \%$ in transductal-LCBDE group $(p=0.573)$. The duration of operation in micro-LCBDE was a little shorter than that in transductal-LCBDE: median 125 (interquartile range 95-173) versus 150 (120-195) min respectively $(p=0.003)$. Postoperative recovery was faster in micro-LCBDE than in transductal-LCBDE, as reflected by a shorter postoperative hospital stay (median 3 versus 5 days, $p=0.002$, respectively). There was also no significant difference in rates of retained stone and recurrence of CBD stone between the groups.

Conclusions: Our findings suggest that micro-LCBDE surgery was effective and safe as transductal-LCBDE surgery in patients with failed transcystic-LCBDE, suggesting that the micro-LCBDE is an acceptable alternative to transductal-LCBDE for failed transcystic-LCBDE. 